\title{
Simulation of the LHCb Electromagnetic Calorimeter response with GEANT4
}
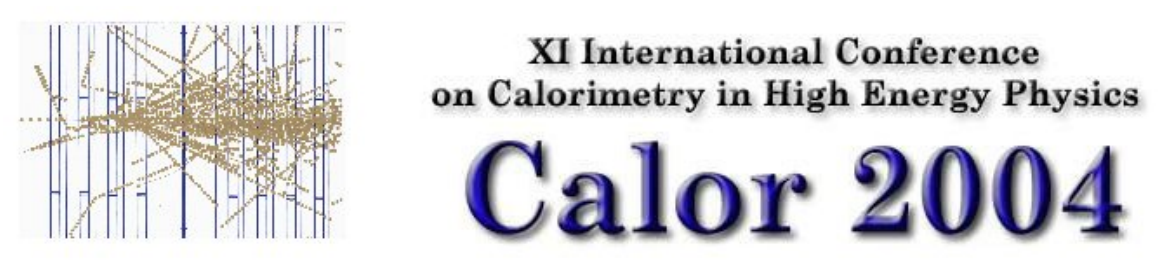

Patrick Robbe (LAL Orsay) for the LHCb Calorimeter Group, 1 April 2004 LHCb
THCP 


\section{Outline}

- Principles and setup of the LHCb ECAL Simulation

- Specific LHCb ECAL Implementations

- Comparison with test beam data 


\section{The LHCb Detector}

\section{ECAL}




\section{The LHCb Electromagnetic Calorimeter}

\section{(ECAL)}

- Lead - Scintillator shashlik detector

- Light collected by WLS fibers

- Readout by PMT at the back of the detector

- Provides fast information for trigger (high $\mathrm{p}_{\mathrm{T}} \gamma, e, \pi^{0}$ )

- Reconstruction of $B$ hadrons with neutral final states $\left(B^{0} \rightarrow K^{*} \gamma, B^{0} \rightarrow \pi^{+} \pi^{-} \pi^{0}, \ldots\right)$

- Identification of $e$ (for reconstruction, tagging, ...) 


\section{ECAL Simulation with GEANT4}

- Simulation of detector response is based on GEANT4 and ECAL simulation is integrated into the entire LHCb simulation software.

- General framework:

- Primary particles (produced by Pythia for the p-p collisions, EvtGen for B decays and from background) are given to GEANT4 for processing.

- GEANT4 is set up with:

$\square$ specific LHCb geometry and material description

$\square$ specific actions to simulate ECAL behaviour

- Execution speed is an issue 


\section{Geometry Setup}

Outer ECAL: 2688 cells (12 $\mathrm{cm} \times 12 \mathrm{~cm}$ )

Middle ECAL: 1792 cells $(6 \mathrm{~cm} \times 6 \mathrm{~cm})$

Inner ECAL: 1584 cells (4 $\mathrm{cm} \times 4 \mathrm{~cm}$ )
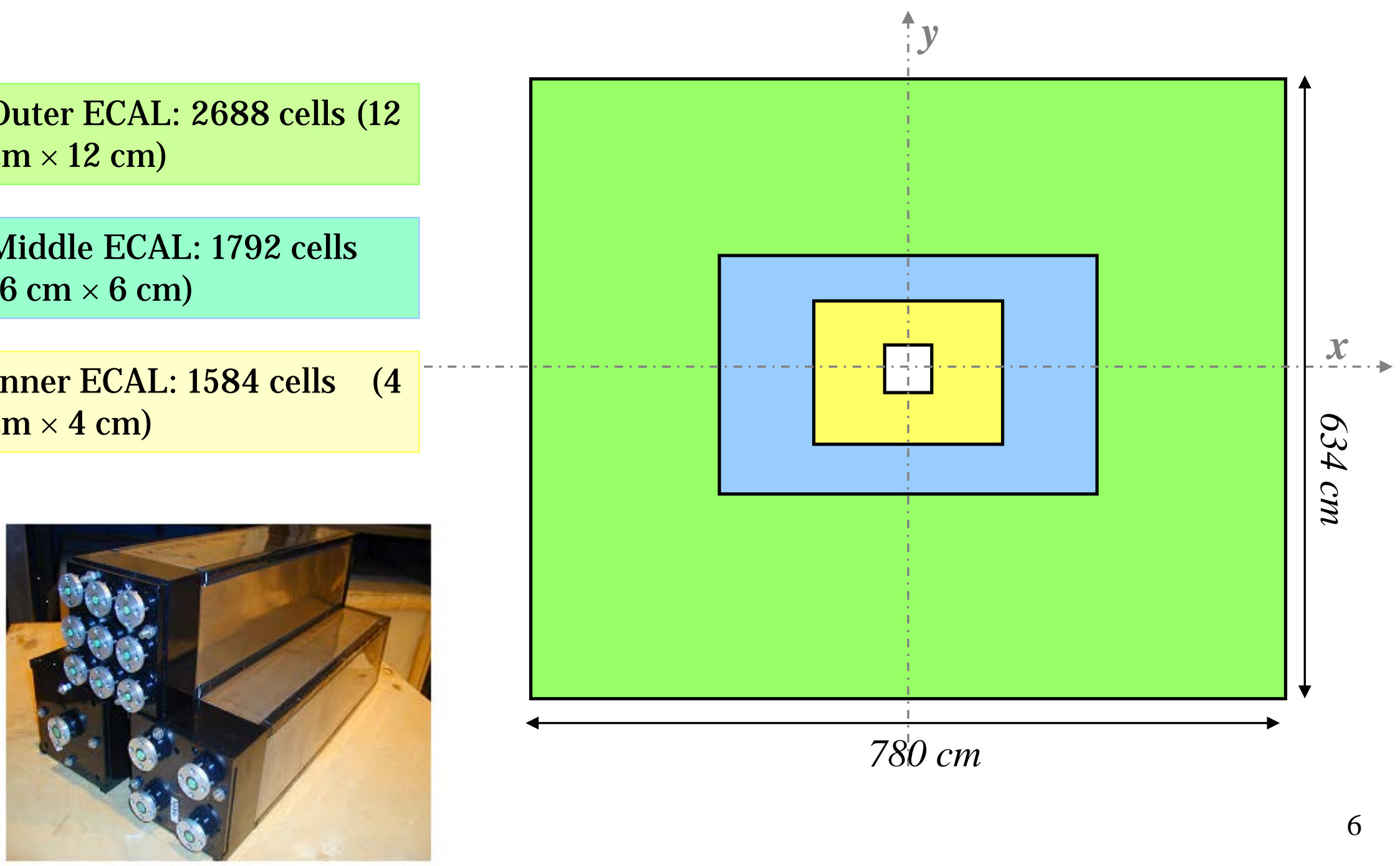


\section{ECAL Outer Module}

- $\underline{\text { Stack: }}$

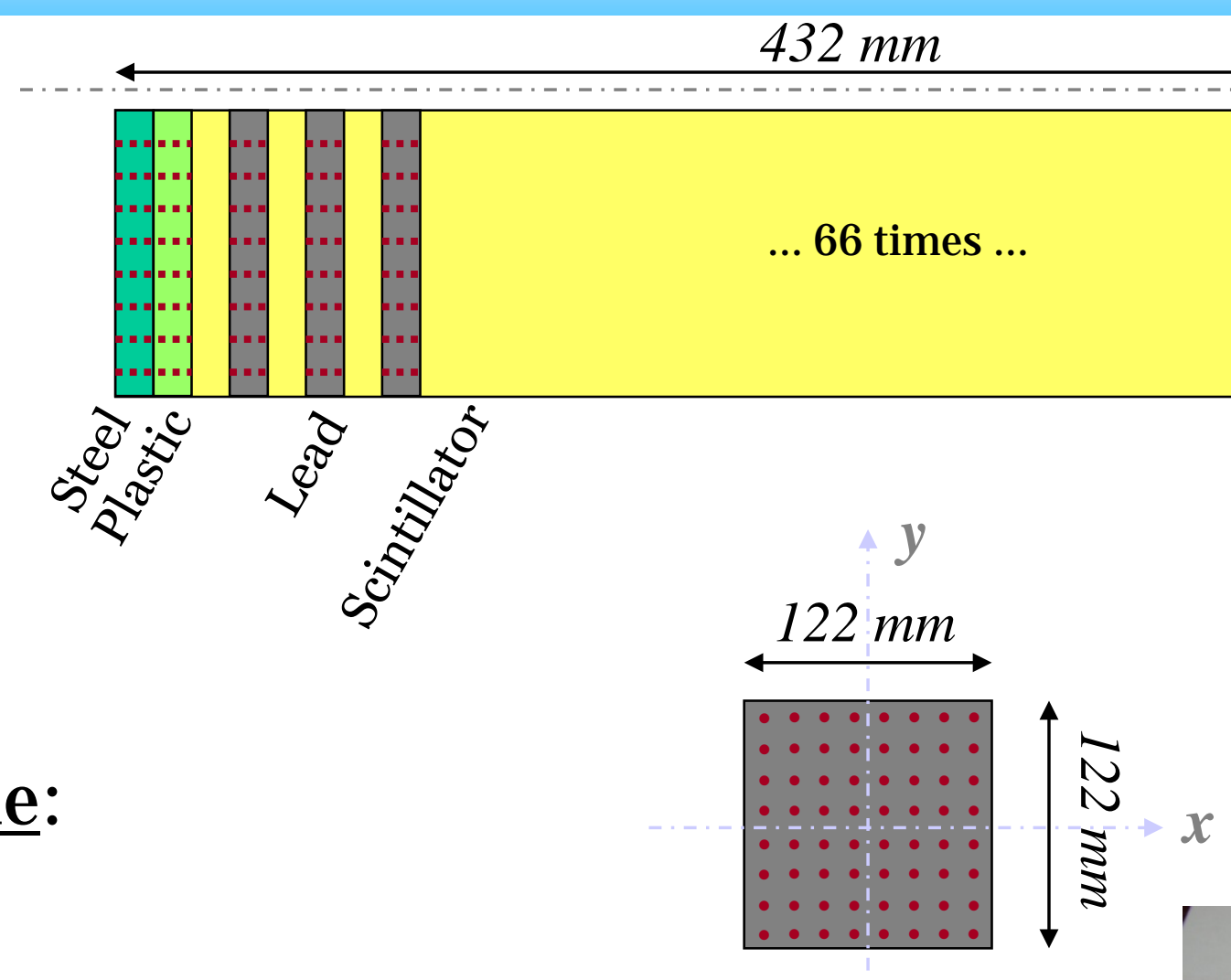

- Detailed and optimized geometry (for example, no fiber in Scintillator, but they are the same material)

- Active material is Scintillator Tile

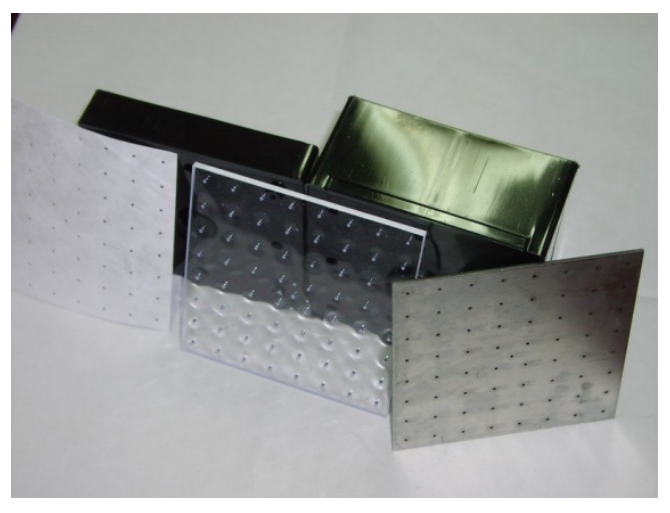




\section{Timing (1)}

- Specific ECAL implementation

- Share each energy deposition in two consecutive 25 ns time bins, according to test beam measurements.

- Simulates signal integration by electronics chain.

- $t_{0}=$ time of arrival of photons at the $z$ position of the maximum of the shower ie 11 cm after ECAL front surface.

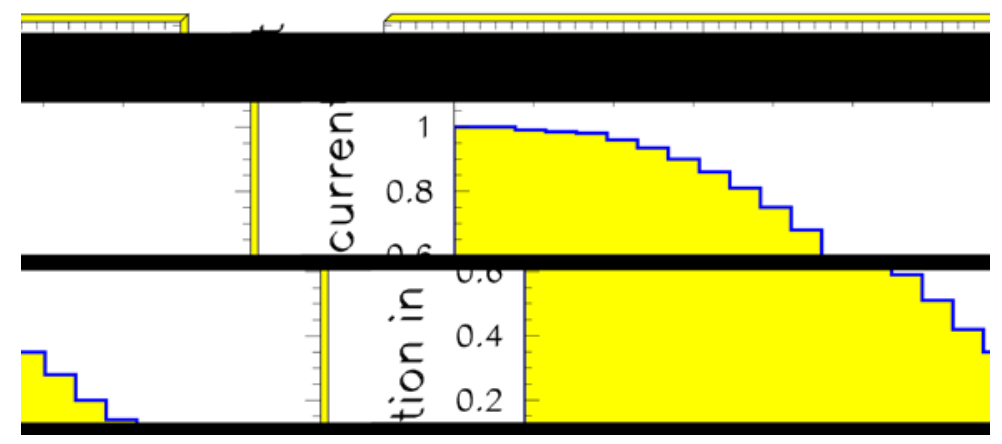




\section{Non Uniformities - Muon Test Beam}

Scan with muons perpendicular to ECAL cell

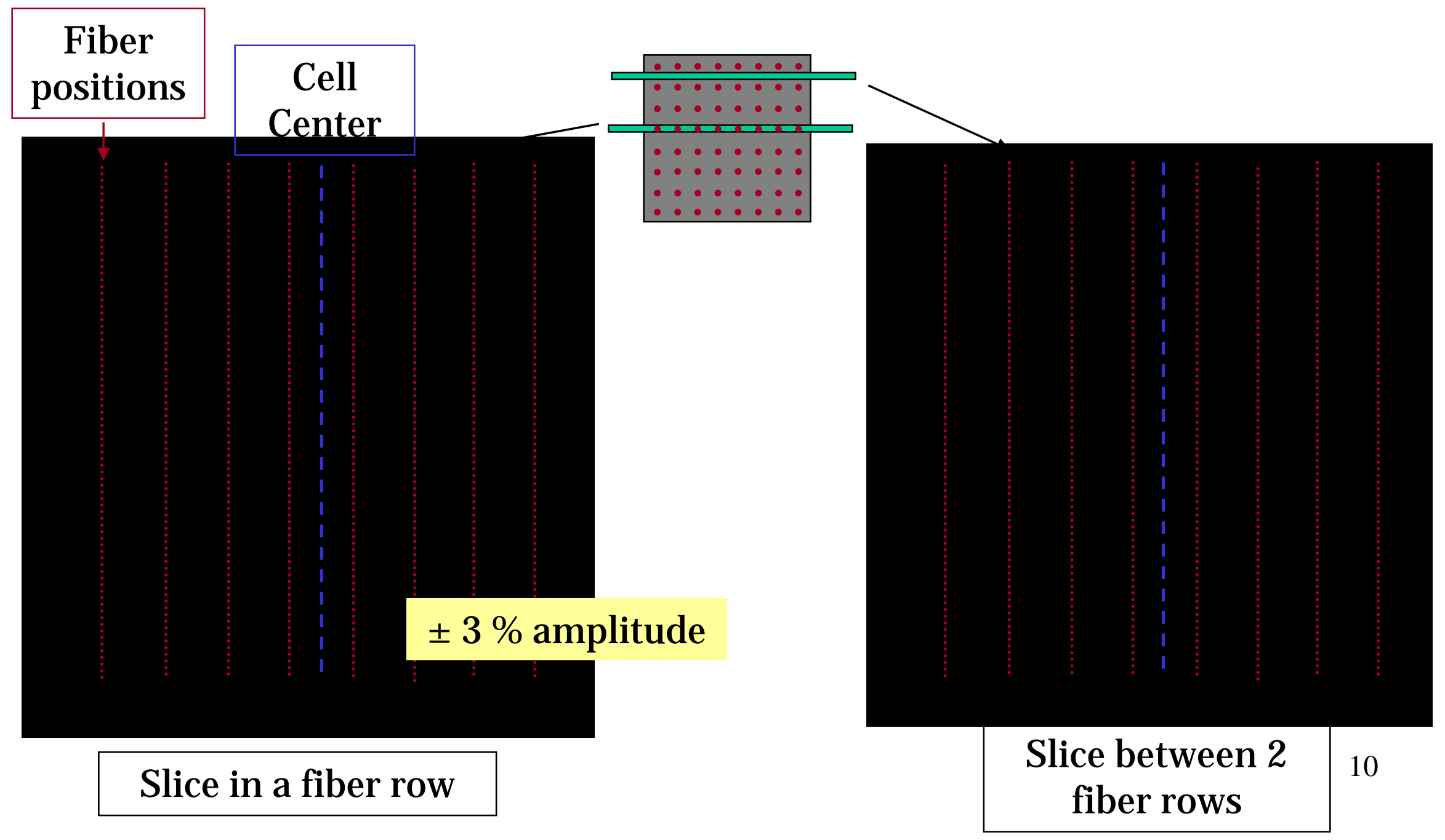




\section{Non Uniformities - Simulation}

- 3 different components:

- Local Non Uniformity:

- $\mathrm{A} \times\left(1-\cos 2 \pi\left(\mathrm{x}-\mathrm{x}_{0}\right) / \mathrm{d}\right) \times\left(1-\cos 2 \pi\left(\mathrm{y}-\mathrm{y}_{0}\right) / \mathrm{d}\right)$

- $\left(\mathrm{x}_{0}, \mathrm{y}_{0}\right)=$ center of the cell

- $\mathrm{d}=$ distance between fibers

- Global Non Uniformity:

- $\mathrm{B} \times\left(\mathrm{x}-\mathrm{x}_{0}+\mathrm{L} / 2\right)^{2} \times\left(\mathrm{y}-\mathrm{y}_{0}+\mathrm{L} / 2\right)^{2}$

- $\mathrm{L}=$ cell size

- Reflection on the edges of the tile (for one side):

- $\mathrm{C} \times \exp \left(-\left|\mathrm{x}-\mathrm{x}_{0}+\mathrm{L} / 2\right| / \mathrm{D}\right)$ 


\section{Non Uniformities - Muon Simulation}

Scan with muons perpendicular to ECAL cell

\section{Cell Fdore}

\section{Cell Center}

$\pm 3 \%$ amplitude
Slice between 2 fiber rows 


\section{Non Uniformities - Electron Test Beam}

Scan with $50 \mathrm{GeV}$ electrons perpendicular to ECAL cell

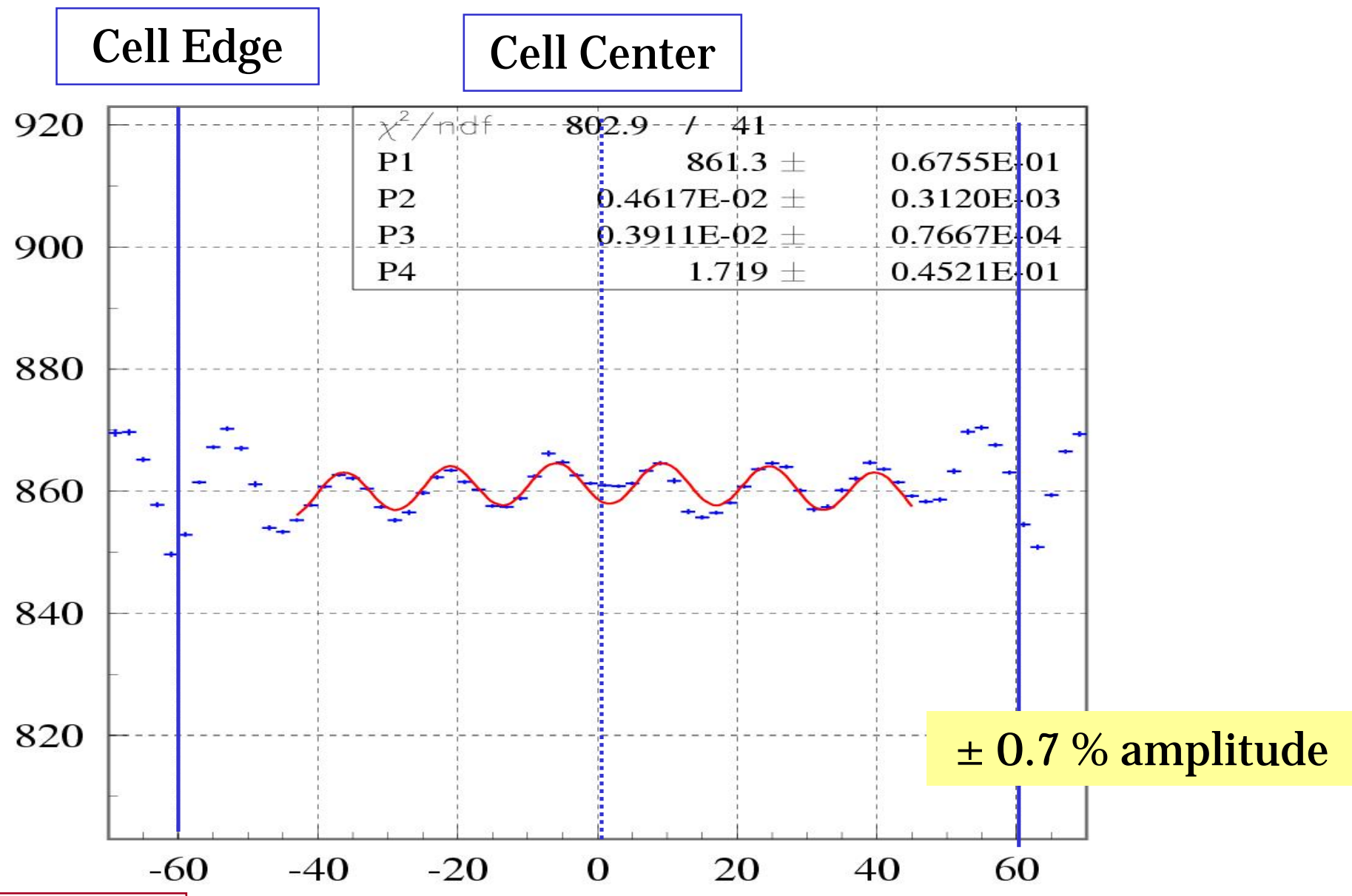

Preliminary results 


\section{Non Uniformities - Electron Simulation}

Scan with $50 \mathrm{GeV}$ electrons perpendicular to ECAL cell

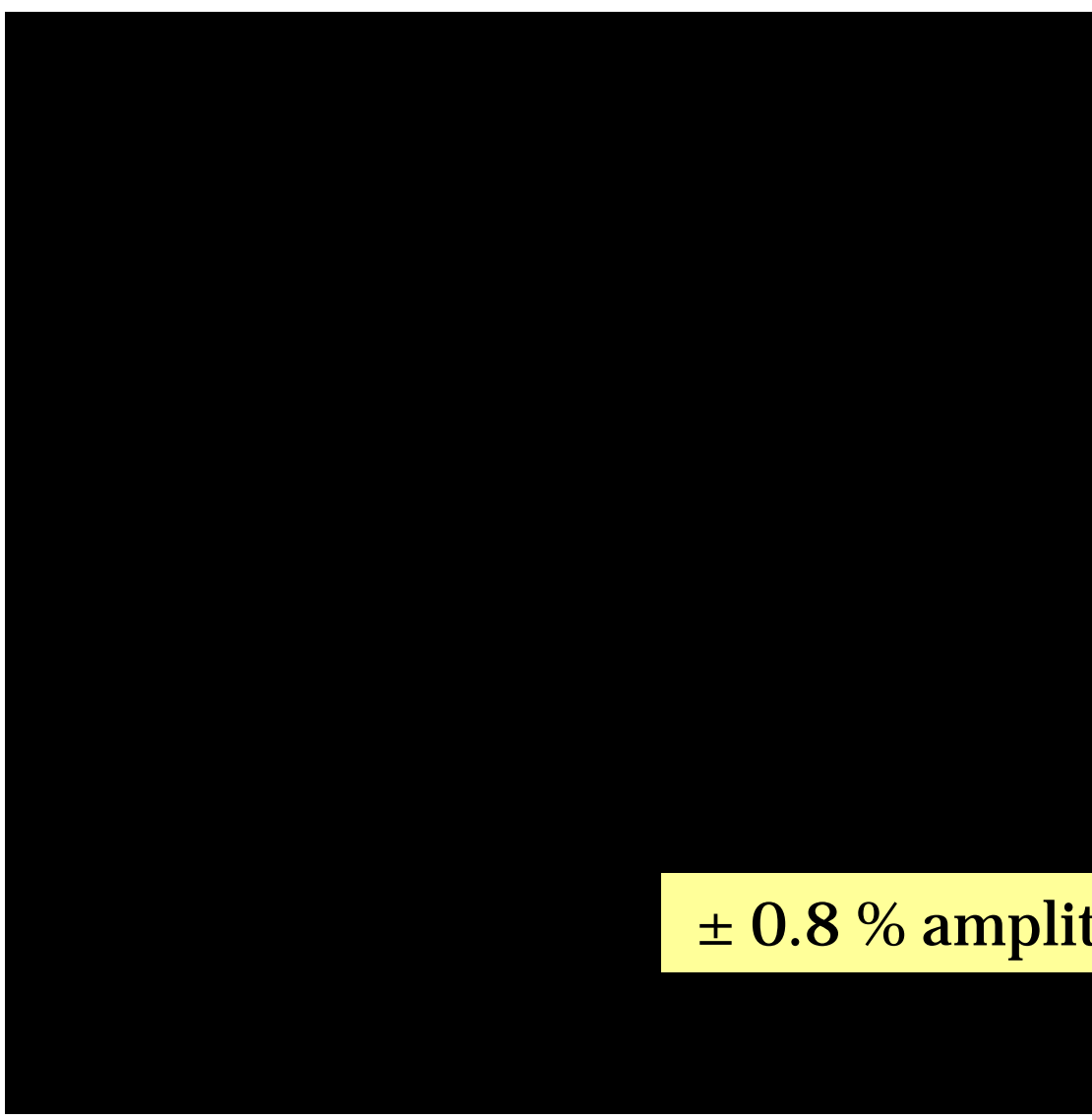

- Preliminary results

- Parameters that reproduce muon test beam data do not reproduce electron test beam data (factor 2 between them)

- This will be studied in more details (including other cell size of ECAL) with test beam this summer 


\section{Resolution - Test Beam}

Electrons perpendicular to the module
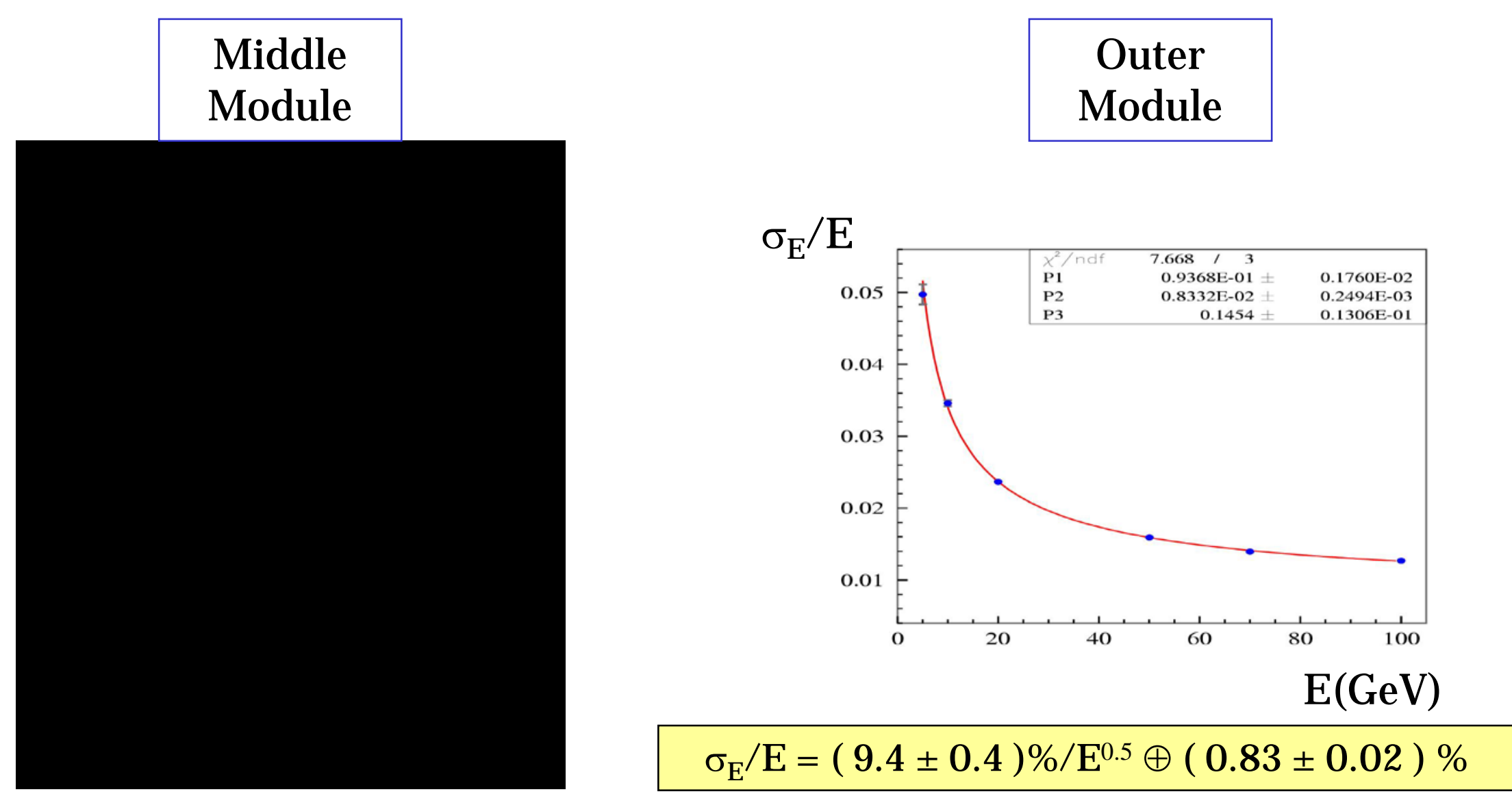

$\sigma_{E} / E=(7.8 \pm 0.3) \% / E^{0.5} \oplus(0.78 \pm 0.05) \%$ 


\section{Resolution - Simulation}

Electrons perpendicular to one Outer Module

\section{Linearity}
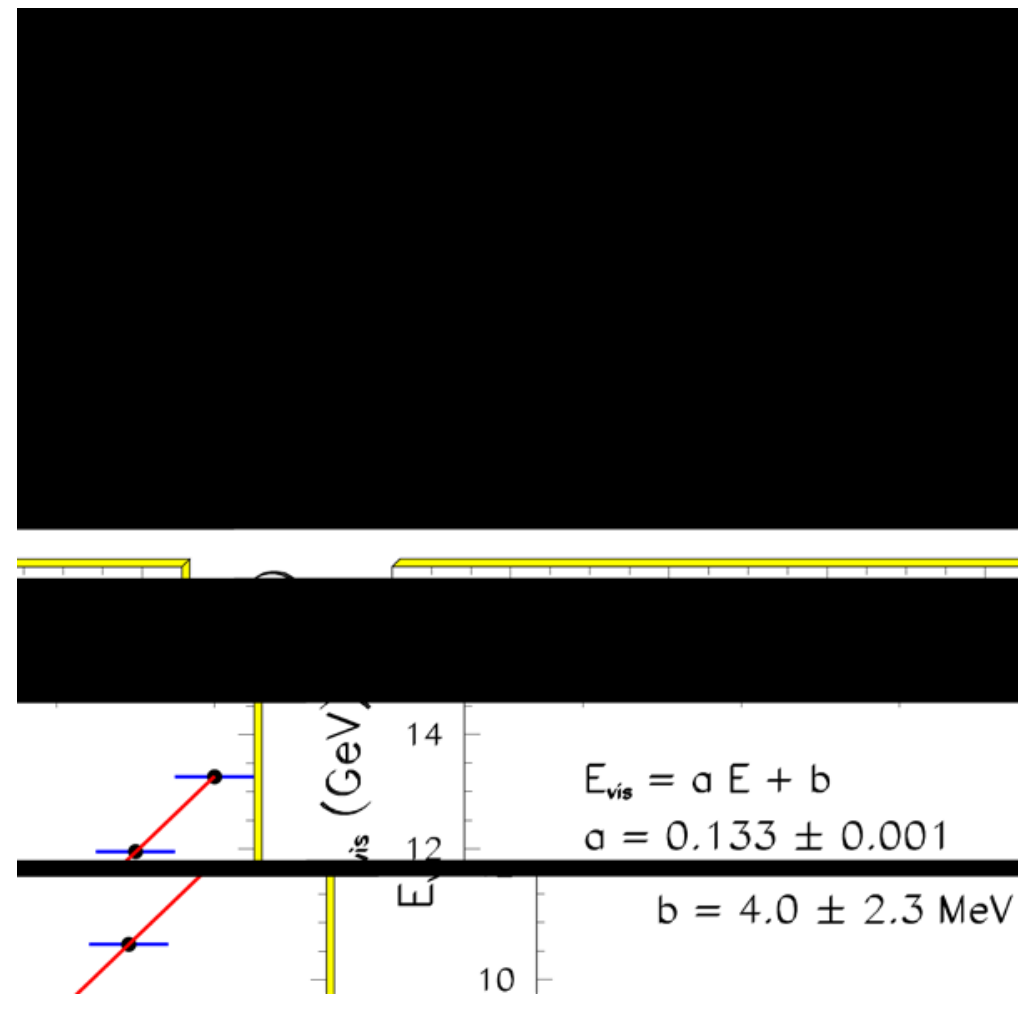

Active $/$ Total $=7.5 \pm 0.1$
Resolution

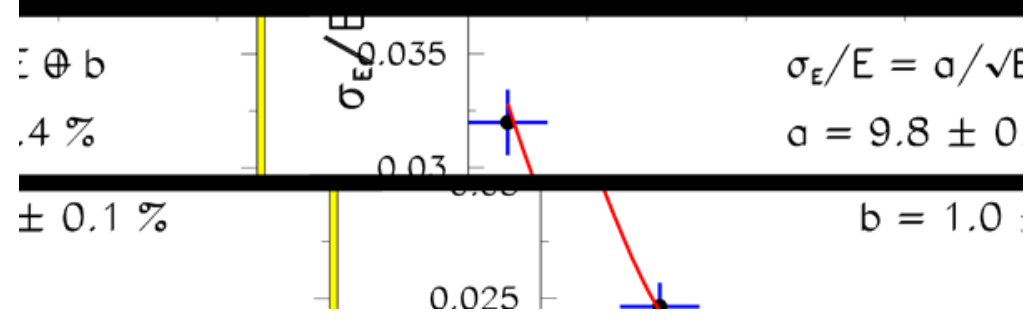

$\sigma_{\mathrm{E}} / \mathrm{E}=(9.8 \pm 0.4) \% / \mathrm{E}^{0.5} \oplus(1.0 \pm 0.1) \%$ 


\section{Conclusions}

- LHCb simulation software contains a realistic simulation of ECAL detector.

- Timing and non uniformities are taken into account in the simulation.

- For non uniformities, more detailed studies with new test beam data will be performed in the near future. 